\title{
Avaliação funcional de idosos atendidos na Atenção Básica
}

\author{
Functional evaluation of elderly attended in Primary Care \\ Evaluación funcional de ancianos atendidos en Atención Primaria
}

\section{Resumo}

Objetivo: Estimar a prevalência de incapacidade funcional para atividades básicas e instrumentais da vida diária e sua associação com características socioeconômicas, demográficas e de saúde em idosos. Metodologia: Foi aplicado questionário semiestruturado para coleta de dados sociodemográficos, foram avaliados através das escalas de KATZ e de LAWTON também sendo avaliada a mobilidade através do TESTE TIME GET UP AND GO TEST. Resultado: O estudo foi composto por 15 homens e 28 mulheres. Tivemos predominância de idosos com faixa etária de 60 a 69 anos e casados. Maior prevalência de baixa escolaridade, aposentados e agricultores. A hipertensão arterial sistêmica foi a doença de maior prevalência. O maior percentual de idosos recebem assistência de médico, enfermagem e dos agentes comunitários de saúde. Na avaliação da escala de Katz, mostram-se independentes. Na escala de Lawton, $87 \%$ dos homens e $86 \%$ das mulheres são independentes. Já quanto ao risco de quedas, $76,7 \%$ dos idosos apresentaram médio risco. Conclusão: Todos os objetivos impostos nesta pesquisa, foram alcançados com clareza e ao observar todos esses achados, seria de suma importância, aplicar um protocolo de exercícios fisioterapêuticos para estes idosos.

Palavras-chave: Envelhecimento; Funcionalidade; Atividades de vida diária; Atenção Básica.

\begin{abstract}
Objectives: to estimate the prevalence of functional incapacity for basic and instrumental activities of daily living and its association with socioeconomic, demographic and health characteristics in the elderly. methodology: a semistructured questionnaire was requested to collect sociodemographic data, obtained through the katz and lawton scales, and mobility was also assessed through the test time get up and go test. results: outcome the study consisted of 15 men and 28 women. we had a predominance of elderly people aged between 60 and 69 years old and married. higher prevalence of low education, retirees and farmers. systemic arterial hypertension was the most prevalent disease. the highest percentage of elderly people is assisted by a doctor, nursing and responsible health agents. in the evaluation of the katz scale, it is shown to be independent. on the lawton scale, $87 \%$ of men and $86 \%$ of women are independent. as for the risk of falls, $76.7 \%$ of the elderly were at medium risk. conclusion: all the objectives imposed in this research were clearly achieved and when observing all these findings, it would be extremely important to apply a physical therapy exercise protocol for these elderly people.
\end{abstract}

Keywords: Aging; Functionality; Activities of daily living; Primary Care.

\section{Resumen}

Objetivos: Estimar la prevalencia de incapacidad funcional para las actividades básicas e instrumentales de la vida diaria y su asociación con características socioeconómicas, demográficas y de salud en los ancianos. Metodología: Se solicitó un cuestionario semiestructurado para la recolección de datos sociodemográficos, obtenidos a través de las escalas KATZ y LAWTON, y también se evaluó la movilidad mediante el TEST TIME GET UP AND GO TEST. Resultado: El estudio consistió en 15 hombres y 28 mujeres. Tuvimos un predominio de personas mayores de entre 60 y 69 años y casadas. Mayor prevalencia de bajos niveles de educación, jubilados y agricultores. La hipertensión arterial sistémica fue la enfermedad más prevalente. El mayor porcentaje de personas mayores es atendido por un médico, enfermeras y agentes sanitarios responsables. En la evaluación de la escala de Katz, se muestra independiente. En la escala de Lawton, el $87 \%$ de los hombres y el $86 \%$ de las mujeres son independientes. En cuanto al riesgo de caídas, el 76,7\% de los 
ancianos tenían un riesgo medio. Conclusión: Todos los objetivos impuestos en esta investigación fueron claramente alcanzados y al observar todos estos hallazgos, sería de suma importancia aplicar un protocolo de ejercicio de fisioterapia para estas personas mayores.

Palabras clave: Envejecimiento; Funcionalidad; Actividades de la vida diaria; Atención Primaria.

\section{Introdução}

O aumento da proporção de idosos não é um fenômeno repentino ou inesperado, pois resulta das transformações demográficas ocorridas nas décadas passadas, motivo pelo qual, na maioria dos países, será um processo severo, com muitas repercussões na saúde pública. com exceção de alguns países africanos, todo mundo está em algum estágio deste processo. não se trata de um fenômeno isolado, sistematicamente está associado a modificações do perfil epidemiológico e das características sociais e econômicas de uma determinada população (Chaimowicz et al, 2013).

"Entende-se o envelhecimento como um processo natural, progressivo e dinâmico, no qual existem alterações morfológicas, funcionais e bioquímicas, que tornam o organismo mais susceptível a agressões e a deformidades.” (Xavier, et al. 2019).

De acordo com miranda, mendes e silva (2016), o envelhecimento da população carrega problemas de saúde que desafiam os sistemas de saúde e de previdência social. os avanços tecnológicos e no sistema de saúde permitiram que a população tivesse acesso a serviços públicos de saúde ou até mesmo, serviços privados adequados, proporcionando uma melhor qualidade de vida nessa fase.

Quando se fala na qualidade de vida (QDV) para o idoso, é importante conhecer as diferentes situações e realidades nas quais esses indivíduos podem estar expostos, visando sempre a boa saúde da pessoa idosa (Dias et al., 2013).

Segundo Martins e Mestre (2014), a QDV é de grande importância na terceira idade, e, ainda mais, em idosos em situação vulnerável, pois o estado de saúde influência de maneira significativa, bem como o seu funcionamento cognitivo e social. Os modelos de QDV vão a partir da satisfação com a vida, o bem-estar social, a modelos baseados em independência, controle, competências sociais e cognitivas.

As alterações fisiológicas do envelhecimento podem causar grande crescimento de limitações ao desempenho de atividades básicas da vida diária, assim, interferindo na funcionalidade da pessoa idosa. Diante disto, ocorrem as alterações visuais; alterações do sistema vestibular; alterações musculoesqueléticas e ósseas; alterações cardiovasculares e neurológicas (Esquenazi et al., 2014).

De acordo com a Classificação Internacional de Funcionalidade (CIF), produzida pela Organização Mundial de Saúde (OMS), em 2003, o termo capacidade reflete a aptidão do indivíduo se ajustar ao ambiente. A funcionalidade está relacionada com as condições de saúde e os fatores contextuais que interage um com o outro. As deficiências em saúde podem modificar a funcionalidade e prejudicar na capacidade da pessoa em interagir com o meio em que vive e causar limitações ao seu desempenho de vida (Paula et al., 2013).

Para Lopes; Santos (2015), a capacidade de executar atividades de vida diária (AVD’s) é um importante indicador de funcionalidade em idosos. Estas são classificadas em atividades básicas de vida diária (ABVD’s), referindo-se ao autocuidado; e atividades instrumentais de vida diária (AIVD’s), que estão relacionadas à vida prática e do cotidiano que este indivíduo vive.

Esta pesquisa justifica-se, pela importância de enfatizar um problema de saúde pública, tendo em vista que a funcionalidade da pessoa idosa é um fator diretamente ligado às alterações fisiológicas do envelhecimento e seus hábitos de vida. A avaliação da funcionalidade da pessoa idosa é de grande utilidade, a fim de observar os déficits funcionais, podendo o fisioterapeuta atuar na prevenção, promoção e reabilitação da saúde da população idosa. 
O objetivo geral da pesquisa foi estimar a prevalência de incapacidade funcional para atividades básicas e instrumentais da vida diária e sua associação com características socioeconômicas, demográficas e de saúde em idosos. E os seguintes objetivos específicos: avaliar a funcionalidade dos idosos atendidos em uma unidade básica de saúde do município de Pocinhos/PB, traçar o perfil sociodemográfico, descrever a assistência ofertada aos idosos pela unidade básica de saúde, identificar as doenças de maior prevalência e verificar diferenças funcionais entre os sexos.

\section{Metodologia}

Neste tópico, estão apresentados os processos metodológicos empregados para a elaboração dessa pesquisa.

\subsection{Tipo de pesquisa}

A presente pesquisa teve um delineamento transversal, tendo o contato em um único momento com os idosos da Unidade Básica de Saúde da Família (UBSF). Apresentou uma abordagem quantitativa, uma vez que esse tipo de pesquisa irá traduzir em números, opiniões e informações para classificá-las e analisá-las, sendo também do tipo exploratória, pois tem a finalidade de proporcionar mais informações sobre o assunto que será investigado (Prodanov \& Freitas, 2013).

\subsection{Cenário}

Esta pesquisa foi realizada na Unidade Básica de Saúde da Família - Rafael Marcone (UBSF) no município de PocinhosPB, endereço: Rua Padre Antônio Galdino, após emissão do Termo de Autorização Institucional.

A UBSF - Rafael Marcone, situada na cidade de Pocinhos compõe em conjunto com as demais a rede de saúde pública do município, com o intuito de oferecer atendimento especializado nos bairros, facilitando o acesso da população a esses serviços, que incluem a marcação de consulta médica, como também fornece cuidados relacionados a odontologia, enfermagem, atuando desde a prevenção de doenças até o tratamento.

\subsection{População e amostra}

A população foi constituída por 439 idosos cadastrados na UBSF - Rafael Marcone. A amostra foi do tipo nãoprobabilística e por acessibilidade, composta por 43 idosos que compareceram nos dias de coleta dos dados da pesquisa, onde estes receberam os devidos esclarecimentos sobre a finalidade e o processo metodológico que o estudo seguiu e aceitaram participar mediante assinatura do TCLE - Termo de Consentimento Livre e Esclarecido. A pesquisa foi realizada nos meses de março e abril de 2019.

\subsection{Critérios de inclusão e exclusão}

Seguiram como critérios de inclusão, idosos iguais ou acima de 60 anos de idade, de ambos os sexos, cadastrados, que frequentam a UBSF e estiveram presentes no período da coleta que aceitaram participar da pesquisa e que assinaram o Termo de Consentimento Livre e Esclarecido - TCLE.

Como critérios de exclusão estão: a recusa explícita, grau de dependência (física ou mental) severa que afastasse o idoso ou o impedisse de se locomover a UBSF. 


\subsection{Instrumentos e procedimentos para coleta de dados}

Como instrumentos para a coleta de dados foram utilizados um questionário semiestruturado a fim de avaliar as características sociodemográficas, clínicas e físicas dos indivíduos participantes do estudo, as escalas de Avaliação Funcional de KATZ e a escala de LAWTON e o TESTE TIME GET UP AND GO TEST (TUG).

Segundo o Ministério da saúde (Brasil, 2007), as escalas que avaliam a funcionalidade do idoso, a escala de Katz foi planejada no intuito de medir a habilidade da pessoa para desempenhar suas atividades cotidianas de maneira independente e assim determinar as necessárias intervenções de reabilitação; e a escala de Lawton tem como foco principal avaliar o desempenho do idoso para realizar as atividades instrumentais e verificar a sua independência funcional. Sendo assim, para complementar as avaliações funcionais, será realizado o teste de TUG, que é utilizado para verificar a mobilidade funcional (Reis et al., 2014).

O TUG, os voluntários foram solicitados a levantar-se de uma cadeira, deambular uma distância de $3 \mathrm{~m}$, virar, retornar e sentar na cadeira novamente, sendo o seu desempenho analisado através da contagem do tempo necessário para realizá-las (Figura 4) (Puerro Neto \& Brito, 2015).

$\mathrm{Na}$ figura abaixo podemos evidenciar como é realizado o seguinte teste.

Figura 1 - Teste de Timed Up and Go (TUGT).

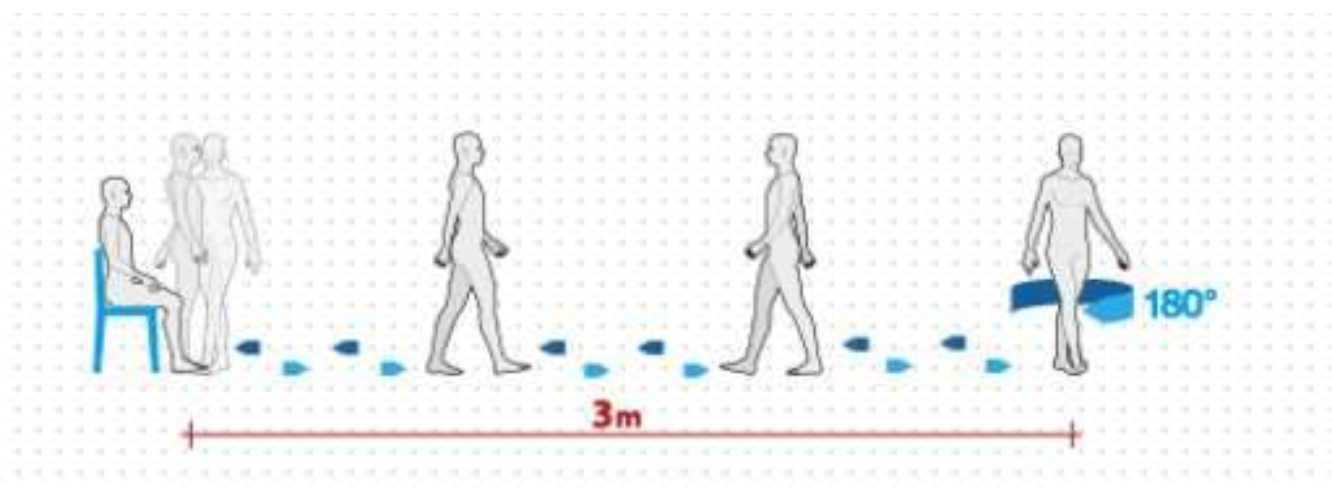

Fonte: Souza (2017).

De acordo com Souza et al. (2017), classificação corresponde: menos de 10 segundos: baixo risco de quedas; 10 a 20 segundos: médio risco de quedas; e acima de 20 segundos: alto risco de quedas.

Para iniciar a pesquisa, o projeto elaborado foi entregue ao responsável pela UBSF onde foi desenvolvida a pesquisa, todos os objetivos e metodologia do estudo foram devidamentes explicados e o mesmo, assinou o Termo de Autorização Institucional.

O projeto de pesquisa foi submetido à apreciação do Comitê de Ética em Pesquisa (CEP) do Centro de Ensino Superior e Desenvolvimento - CESED. Após aprovação e autorização do mesmo sob CAEE: 04915118.8.0000.5175 (ANEXO D), foi iniciada a pesquisa.

Os idosos foram convidados verbalmente a participar da pesquisa, no dia que ocorreu o programa HIPERDIA e saúde mental na UBSF, e os que se enquadraram nos critérios de inclusão e exclusão, foram orientados a respeito dos objetivos e métodos utilizados na pesquisa, quanto ao sigilo e preservação das informações a serem colhidas, bem como informados que os mesmos não teriam gastos com a mesma e que seriam voluntários sem remuneração.

O Hiperdia é um programa que no intuito de fornecer um melhor acompanhamento e aumentar a prevenção dos pacientes com DM e HAS, o Ministério da Saúde inseriu no Brasil, no ano de 2002. Através dele, é realizado o cadastro e acompanhamento dos pacientes, como também a entrega de medicamentos para o tratamento para estas patologias (SILVA; SUTO; COSTA, 2015). 
Logo após todos os esclarecimentos acerca da pesquisa, leitura do Termo de Consentimento Livre e Esclarecido (TCLE) e os sujeitos tendo atendido a todos os critérios, os participantes foram convidados a assinar o TCLE, o qual foi lido antes do início da coleta de dados para esclarecimentos sobre a pesquisa e suas questões éticas e entregue uma via para cada participante do estudo.

Depois da assinatura do TCLE os idosos responderam primeiramente ao questionário sociodemográfico, e em seguida foram avaliados através da escala de Katz (ANEXO B), Lawton e TUGT visando avaliar a funcionalidade.

A coleta dos dados contem informações sociodemográficas, sobre a assistência ofertada aos idosos pela UBSF, as doenças de maior prevalência, também foram realizadas as avaliações através das escalas de avaliação funcional: Lawton e Katz e o teste de TUGT para a avaliação do risco de quedas.

\subsection{Processamento e análise de dados}

Todos os dados foram processados, armazenados e analisados em um banco de dados em formato de planilhas no software Microsoft Office Excel versão 2016. A análise dos dados foi realizada através do software Statistical Package for the Social Scienses (SPSS) versão 24.

\subsection{Apresentação dos resultados}

Os resultados foram apresentados através de gráficos e tabelas elaboradas utilizando o software Microsoft Office versão 2016.

\subsection{Aspectos éticos}

A pesquisa seguiu a recomendação da Comissão Nacional de Ética em Pesquisa - CONEP, expresso a resolução 466/2012 do Conselho Nacional de Saúde (CNS), na qual aprova as diretrizes e normas regulamentadoras de pesquisas em que envolve seres humanos, baseando nos principios básicos da bioética: autonomia, beneficência, não-maleficência e justiça.

Antes de coletar os dados, o projeto foi submetido à aprovação pelo responsável da UBSF, mediante autorização institucional. Após isto, o projeto foi submetido à apreciação do Comitê de Ética do CESED e só será iniciada a pesquisa após a emissão do parecer favorável.

O pesquisador responsável assinou o Termo de Compromisso do Pesquisador.

\section{Resultados e Discussão}

No tempo estimado de dois meses, foram avaliados 43 idosos de uma população de 439 idosos cadastrados na UBSF Rafael Marcone. As tabelas e gráficos apresentadas referem-se aos resultados obtidos no questionário sociodemográfico, nas escalas de avaliação da funcionalidade de Katz e Lawton e do teste de Timed Up and Go (TUG). Os dados foram armazenados e tratados no SPSS versão 24 para Windows. As análises foram realizadas por meio da estatística descritiva onde foram expostos os valores absolutos, relativos.

\subsection{Caracterização do perfil sociodemográfico, ocupacional e profissional da amostra}

Nesse tópico, serão apresentados os dados sociodemográficos referente a esse estudo. A tabela a seguir, aponta o perfil sociodemográfico da população estudada, onde pode-se verificar variáveis como sexo, faixa etária, cor, estado civil, escolaridade, número de filhos, renda, entre outras. 
Tabela 1 - Perfil sociodemográfico dos indivíduos estudados.

\begin{tabular}{|c|c|c|c|}
\hline Variáveis & & n (43) & $\%$ \\
\hline \multirow[t]{2}{*}{ Sexo } & Masculino & 15 & 34,9 \\
\hline & Feminino & 28 & 65,1 \\
\hline \multirow[t]{3}{*}{ Faixa etária } & $60-69$ & 26 & 60,5 \\
\hline & $70-79$ & 13 & 30,2 \\
\hline & $80-89$ & 4 & 9,3 \\
\hline \multirow[t]{2}{*}{ Cor } & Branca & 33 & 76,7 \\
\hline & Parda & 10 & 23,3 \\
\hline \multirow[t]{4}{*}{ Estado civil } & Solteiro & 3 & 7,0 \\
\hline & Casado & 27 & 62,8 \\
\hline & Divorciado/Separado & 5 & 11,6 \\
\hline & Viúvo & 8 & 18,6 \\
\hline \multirow[t]{6}{*}{ Escolaridade } & Não escolarizado & 3 & 7,0 \\
\hline & $\begin{array}{l}\text { Fundamental } \\
\text { incompleto } \\
\text { Fundamental completo }\end{array}$ & $\begin{array}{l}30 \\
2\end{array}$ & $\begin{array}{l}69,7 \\
4,7\end{array}$ \\
\hline & Médio incompleto & - & 0 \\
\hline & Médio completo & 4 & 9,3 \\
\hline & Superior incompleto & 1 & 2,3 \\
\hline & Superior completo & 3 & 7,0 \\
\hline \multirow[t]{4}{*}{ Número de filhos } & Nenhum & 4 & 9,3 \\
\hline & 1 a 3 filhos & 13 & 30,2 \\
\hline & 4 a 6 filhos & 20 & 46,5 \\
\hline & 7 a 9 filhos & 6 & 14 \\
\hline \multirow[t]{2}{*}{ Chefe de família } & Sim & 35 & 81,4 \\
\hline & Não & 8 & 18,6 \\
\hline \multirow[t]{3}{*}{ Renda } & Até 1 salário & 25 & 58,1 \\
\hline & De 2 a 3 salários & 14 & 32,6 \\
\hline & De 4 ou mais salários & 4 & 9,3 \\
\hline \multirow[t]{5}{*}{ Com quem vive* } & Esposo (a) & 26 & 60,4 \\
\hline & Filhos & 22 & 51,1 \\
\hline & Netos & 6 & 13,9 \\
\hline & Genros-Noras & 2 & 4,7 \\
\hline & Sozinho & 8 & 18,6 \\
\hline
\end{tabular}

*O valor de n não corresponde a amostra e o valor relativo ultrapassa $100 \%$ pois os indivíduos podiam afirmar mais de uma resposta. Fonte: Dados da pesquisa (2019).

Seguindo o contexto histórico da população, na tabela 2, pode-se imprimir o perfil ocupacional e profissional dos indivíduos 
Research, Society and Development, v. 11, n. 1, e12011124968, 2022

(CC BY 4.0) | ISSN 2525-3409 | DOI: http://dx.doi.org/10.33448/rsd-v11i1.24968

Tabela 2 - Perfil ocupacional e profissional dos indivíduos estudados.

\begin{tabular}{|c|c|c|c|}
\hline Variáveis & & $\begin{array}{l}\mathrm{n} \\
(43)\end{array}$ & $\%$ \\
\hline \multirow[t]{5}{*}{ Ocupação } & Aposentado & 38 & 88,4 \\
\hline & Empresário & 1 & 2,3 \\
\hline & Funcionário público & 1 & 2,3 \\
\hline & Do lar & 2 & 4,7 \\
\hline & Pensionista & 1 & 2,3 \\
\hline \multirow[t]{17}{*}{ Profissão } & Agricultor & 20 & 46,6 \\
\hline & Do lar & 4 & 9,4 \\
\hline & Professor & 4 & 9,4 \\
\hline & Merendeira & 2 & 4,7 \\
\hline & Auxiliar Serviços Gerais & 1 & 2,2 \\
\hline & Auxiliar administrativo & 1 & 2,3 \\
\hline & Auxiliar de enfermagem & 1 & 2,3 \\
\hline & Cabeleireiro & 1 & 2,3 \\
\hline & Carpinteiro & 1 & 2,3 \\
\hline & Gráfico & 1 & 2,3 \\
\hline & Industrial & 1 & 2,3 \\
\hline & Operador de máquina & 1 & 2,3 \\
\hline & Pedreiro & 1 & 2,3 \\
\hline & Empresário & 1 & 2,3 \\
\hline & Funcionário público & 1 & 2,3 \\
\hline & Costureiro & 1 & 2,3 \\
\hline & Comerciante & 1 & 2,3 \\
\hline
\end{tabular}

Fonte: Dados da pesquisa (2019).

\subsection{Perfil epidemiológico, assistencial e de hábitos de vida}

Neste tópico, serão apresentados os dados da tabela referente ao perfil epidemiológico dos idosos estudados.

A seguinte amostra, evidencia as doenças de maior prevalência na população e a assistência ofertada pelo centro de saúde do munícipio (Tabela 3). 
Research, Society and Development, v. 11, n. 1, e12011124968, 2022

(CC BY 4.0) | ISSN 2525-3409 | DOI: http://dx.doi.org/10.33448/rsd-v11i1.24968

Tabela 3 - Perfil epidemiológico e assistencial da amostra estudada.

\begin{tabular}{llll}
\hline Variáveis & & $\mathrm{n}(43)$ & $\%$ \\
\hline Tem doença crônica diagnosticada & Sim & 33 & 76,7 \\
Doenças crônicas diagnosticadas* & Não & 10 & 23,3 \\
& HAS & 25 & 58,1 \\
& Diabetes & 8 & 18,6 \\
& Depressão & 4 & 9,3 \\
& Hipertireoidismo & 4,6 & 4,6 \\
& Angina & 2 & 2,3 \\
& Câncer & 2 & 2,3 \\
& Trombose & 1 & 2,3 \\
& Glaucoma & 1 & 2,3 \\
& Enxaqueca & 1 & 2,3 \\
Faz uso medicação & Artrose & 1 & 2,3 \\
& Epilepsia & 1 & 2,3
\end{tabular}

*O valor de n não corresponde a amostra pois os indivíduos podiam afirmar mais de uma opção. Fonte: Dados da pesquisa (2019).

A Tabela 4 demonstra através dos estudos os hábitos de vida dos indivíduos, podendo proporcionar um conhecimento mais amplo da população.

Tabela 4 - Hábitos de vida da amostra estudada.

\begin{tabular}{llll}
\hline Etilismo & Sim & 7 & 16,3 \\
Tabagismo & Não & 36 & 83,7 \\
& Sim & 4 & 9,3 \\
Prática de Atividade física & Não & 39 & 90,7 \\
& Sim & 20 & 46,5 \\
Frequência de atividade física & Não & 23 & 53,5 \\
& Todo dia & 16 & 37,2 \\
& Três vezes por semana & 3 & 6,9 \\
Qual atividade física & Às vezes & 1 & 2,3 \\
& Caminhada & 17 & 39,5 \\
& Esteira & 2 & 4,6 \\
& Pilates & 1 & 2,3
\end{tabular}

Fonte: Dados da pesquisa (2019). 


\subsection{Funcionalidade}

$\mathrm{Na}$ (Tabela 5) os idosos desta pesquisa mostraram-se independentes para quase todas as atividades. Banho (100\%), vestir-se (100\%), higiene (100\%), transferência (100\%), continência (86\%) e alimentar-se (100\%).

Tabela 5 - Descrição da funcionalidade referente à escala de Katz.

\begin{tabular}{llll}
\hline Variáveis & & $\mathrm{n}$ & (43) \\
\hline Banho & Independente & 43 & 100 \\
& Dependente & - & 0 \\
Vestir-se & Independente & 43 & 100 \\
& Dependente & - & 0 \\
Higiene & Independente & \multicolumn{2}{l}{100} \\
& Dependente & 43 & 0 \\
Transferência & Independente & \multicolumn{2}{c}{0} \\
& Dependente & 43 & 100 \\
Continência & Independente & \multicolumn{2}{c}{0} \\
& Dependente & 37 & 86,0 \\
Alimentar-se & Independente & 6 & 14,0 \\
& Dependente & 43 & 100 \\
\hline
\end{tabular}

Fonte: Dados da pesquisa (2019).

A Tabela 6, trás dados relevantes com relação a funcionalidade dos idosos avaliados, mostrando o grau de independência e dependência das AVD's.

Tabela 6 - Descrição da funcionalidade referente à escala de Lawton.

\begin{tabular}{lllll}
\hline Variáveis & Homem & \multicolumn{3}{c}{ Mulher } \\
\cline { 2 - 5 } Independente & $\mathbf{n}$ & $\%$ & $\mathbf{n}$ & $\%$ \\
Dependente parcial & 13 & 87 & 24 & 86 \\
Dependente & 2 & 13 & 4 & 14 \\
\hline
\end{tabular}

Fonte: Dados da pesquisa (2019).

O resultado referente ao teste de TUGT segue demonstrado na tabela 7, e quantifica através dos segundos, o tempo percorrido pelo indivíduo no trajeto realizado.

Tabela 7 - Medidas descritivas referentes ao TUGT.

\begin{tabular}{llll}
\hline Variáveis & & $\mathrm{n}$ & $\%$ \\
\hline Segundos & $<10$ segundos & 10 & 23,3 \\
& 10 a 20 segundos & 33 & 76,7 \\
\hline
\end{tabular}

Fonte: Dados da pesquisa (2019).

\subsection{Diferenças funcionais entre os sexos}

O gráfico a seguir, informa os dados em porcentagem referente ao estudo realizado através da escala de Katz. 
Gráfico 1 - Porcentagem referente à Escala de Katz, por sexo.

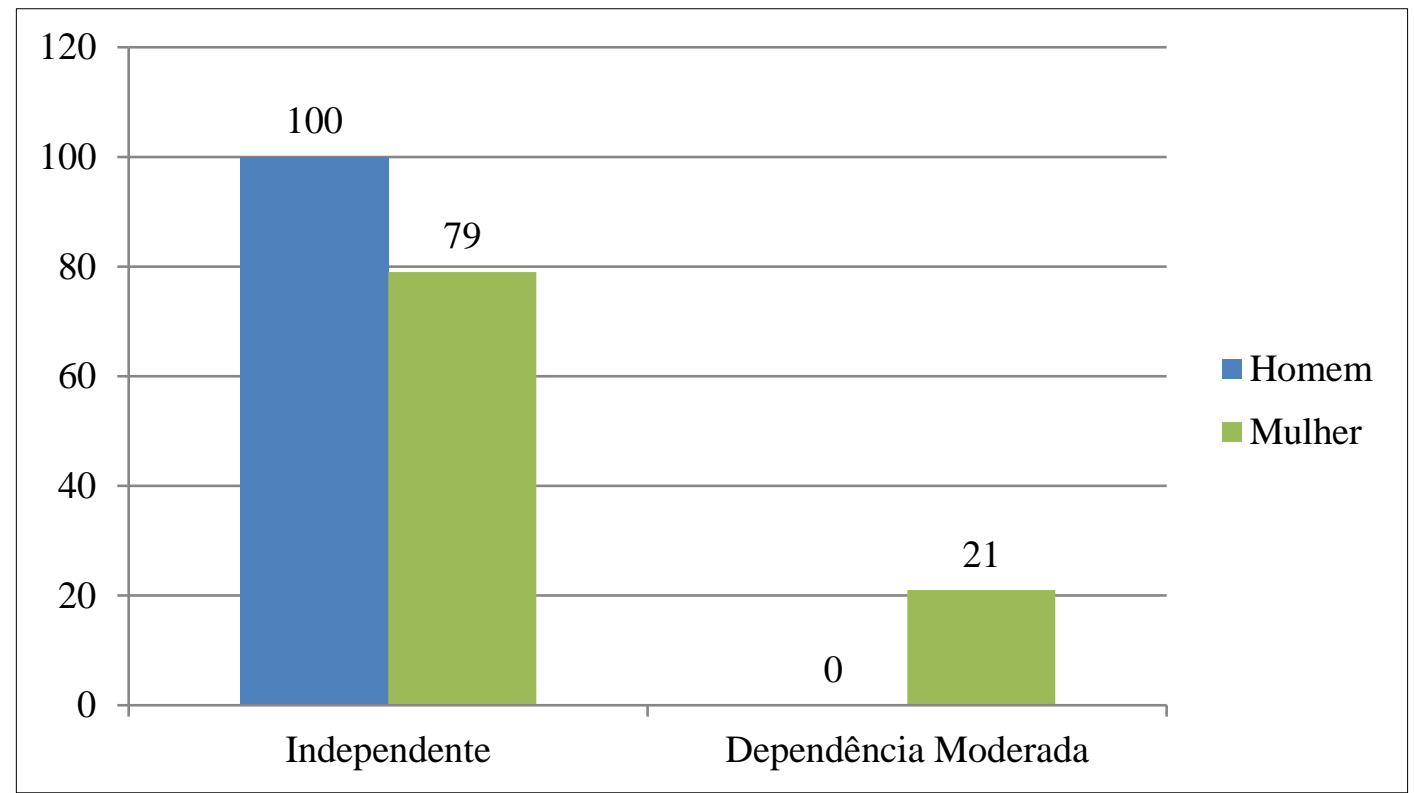

Fonte: Dados da pesquisa (2019).

O segundo gráfico apresenta os resultados obtidos pela avaliação feita utilizando a escala de Lawton.

Gráfico 2 - Porcentagem referente à Escala de Lawton, por sexo.

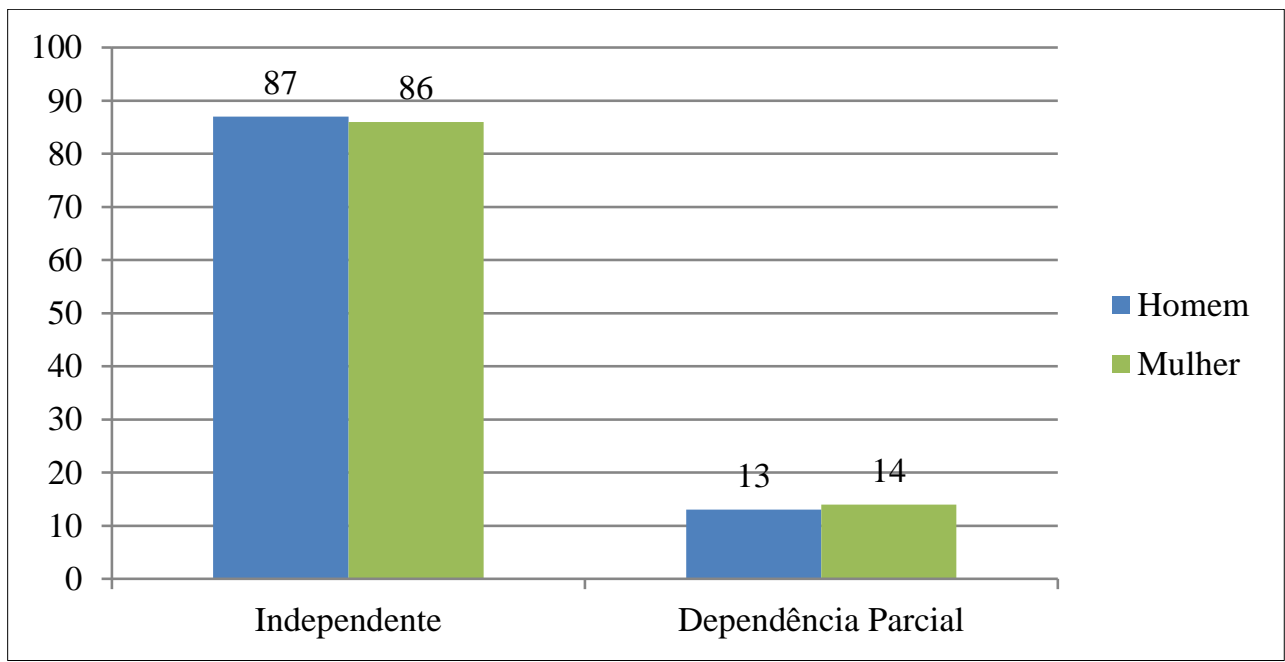

Fonte: Dados da pesquisa (2019).

No gráfico abaixo, pode-se evidenciar a porcentagem referente ao risco de quedas em idosos, utilizando o teste de TUGT. 
Gráfico 3 - Porcentagem referente ao Teste de TUGT para risco de quedas, por sexo.

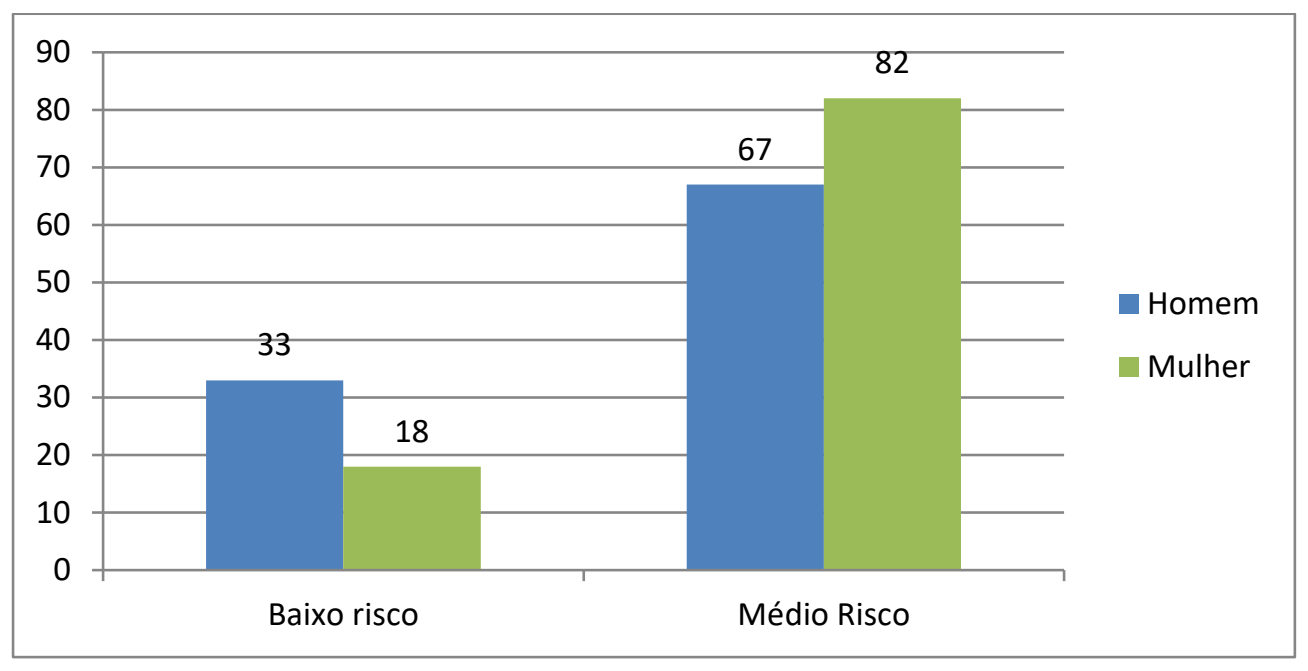

Fonte: Dados da pesquisa (2019).

Conforme apresentado na Tabela 1, este estudo foi composto por 15 homens e 28 mulheres. Os idosos dessa população tem predominância de $(\mathrm{n}=28)(65,1 \%)$ sendo do sexo feminino, no geral, estão na faixa etária entre 60 a 69 anos de idade, a maioria $(n=33)(76,7 \%)$ são de cor branca, a maior parte desses idosos são casados $(n=27)(62,8 \%)$. Em relação à escolaridade $(n=30)(69,7 \%)$ apresentaram baixa escolaridade, tendo apenas o ensino fundamental incompleto. Com um percentual de $(90,7 \%)$ a maioria dessas pessoas tem filhos, $(58,1 \%)$ apresentaram renda familiar baixa, de até um salário mínimo. (60,4\%) desses idosos moram com alguém e $(18,6 \%)$ relataram morar sozinhos.

A baixa escolaridade do idoso reflete a necessidade que estes, moradores de cidades do interior, tiveram em parar seus estudos para trabalharem e ter o sustendo do lar. No estudo realizado por Barreto et al. (2017), com 86 idosos participantes, a idade dos mesmos foi entre 60 e 90 anos, com a média de idade de 68,05 anos. Foi evidenciado que, o nível de escolaridade dos idosos foi classificado como baixo, sendo $(\mathrm{n}=44)(83,01 \%)$ possuem o ensino fundamental incompleto. Então, o número de idosos analfabetos e de baixa escolaridade na Região Nordeste do Brasil é prevalente. Isto também é um fator associado à baixa renda dos idosos, que são aposentados pela agricultura.

Segundo Cazella et al. (2016) “As agricultoras e agricultores acessam a aposentadoria por idade, respectivamente, aos 55 e 60 anos, ficando ainda assegurados os auxílios doença e maternidade”.

Referente à quantidade de filhos, ocorreu no Brasil uma queda da natalidade sendo em ambiente de liberdade de escolha, onde teve começo nas camadas mais abundantes e educadas da sociedade, para então se estender progressivamente a toda a população brasileira. Isto decorre do fato de que as mulheres de hoje, estão mais ocupadas, com estudos e trabalhos (Alves, 2014).

Evidencia-se nesse estudo que $(18,6 \%)$ dos idosos entrevistados, moram sozinhos e $(51,1 \%)$ moram com filhos. Observa-se que os idosos de hoje apresentam um percentual maior que 4 filhos por casal. Foi constatado que no ano de 1950 , existia 9 milhões de crianças que tinham de 0 a 4 anos de idade. Este representava $(16,6 \%)$ da população total, enquanto havia 2,6 milhões de idosos com 60 anos ou mais, representando somente $(4,8 \%)$ da população brasileira. No ano de 2030 , decorrente do decrescimento do número médio de filhos das mulheres, a quantidade de crianças de 0 a 4 anos deve ter um decréscimo para 13,3 milhões, representando apenas (6\%) da população. A de idosos deve chegar a 41,6 milhões de pessoas, representando $(18,7 \%)$ da população do Brasil (Alves, 2014).

Segundo Silva e Prá (2014), o Brasil terá um número menor de adultos e uma maior população de idosos. Então, com a redução no número de filhos, estimando uma média de um filho por casal, é possível evidenciar um futuro em que idosos ficarão 
aos cuidados de um único filho, que provavelmente será responsável por prover os cuidados necessários ao pai e a mãe (dois idosos), o que causará uma sobrecarga para este e também para os sistemas de proteção social. Visto isso, observa-se que os idosos de hoje apresentam um número maior de filhos, um fato que vem ocorrendo em menor quantidade nos adultos jovens de acordo com os estudos, evidenciando futuros idosos com menor número de filhos.

Dos idosos avaliados, referente aos dados da Tabela 2 , foi analisado que $(88,4 \%)$ da população são aposentados. De acordo com Cockell (2014), os brasileiros com 65 anos ou mais, 3,1 milhões (22,5\%) ainda trabalham, mas, é evidenciado que a grande maioria são aposentados, constituindo $(74,7 \%)$ e parte da renda desses idosos é proveniente dos benefícios da previdência social, tonando assim, para muitos o único sustento para desses idosos.

Os idosos da cidade de Pocinhos apresentam a profissão de agricultor $(46,6 \%)$ como a mais prevalente e durante a entrevista a maioria (69,7\%) apresentaram baixa escolaridade (Tabela 2). Segundo Busato, et al. (2014), em seu estudo, com 60 idosos, $(95,0 \%)$ dos idosos apresentaram baixa escolaridade, com ensino fundamental incompleto, e com isto, o índice baixo de escolaridade da maior parte dos pesquisados provavelmente se explica pela dificuldade de acesso ao sistema formal de educação, quando os mesmos estavam em idade escolar. Assim, o mesmo relata que esta evidência pode contribuir para uma maior vulnerabilidade e para uma auto percepção negativa de saúde desse grupo, devido a limitação das possibilidades do idoso para lidar com as peculiaridades que estão propostas nesta etapa da vida.

Os dados apresentados na (Tabela 3) evidenciam que dos 43 idosos entrevistados (76,7\%) apresentam alguma doença crônica e $(58,1 \%)$ apresentaram hipertensão arterial sistêmica como a doença de maior prevalência dessa população e $(18,6 \%)$ apresentaram Diabetes. Segundo Costa et al. (2017), o aumento da prevalência de doenças crônicas no país, especialmente a hipertensão arterial, diabetes, artrite/artrose e depressão é resultado do rápido e crescente processo de envelhecimento da população brasileira nos últimos anos.

A pirâmide etária brasileira se encontra em transição com aumento do número de idosos. O envelhecimento apresenta relação direta com a alta incidência das patologias crônicas não transmissíveis e estas, vem sofrendo destaque. As DCNT's de destaque na saúde pública correspondem as Doenças Cardiovasculares, o câncer, o Diabetes Mellitus, e as Doenças Respiratórias Crônicas, ganhando destaque em vários estudos (Silva, et al. 2015).

Foi constatado que o maior percentual de idosos recebe assistência de médico, da enfermagem e dos agentes comunitários de saúde.

Sendo uma das ações propostas para o envelhecimento bem-sucedido é a educação em saúde. Esta é intrínseca às práticas de saúde, onde o seu valor vem sendo reconhecido como dimensão essencial do cuidado em saúde. (SILVA, et al. 2014). "O diagnóstico de uma doença crônica implica mudanças importantes na vida de seus portadores. A partir dele, os pacientes passam a necessitar de um cuidado integral, envolvendo aspectos biológicos, sociais, econômicos e psicológicos, ocasionando uma percepção de pouco controle acerca da própria vida." (Magri, et al. 2020)

No intuito de diminuir a quantidade de hospitalizações e ter o acompanhamento e o tratamento adequado e eficaz na atenção básica para as pessoas necessitadas de tratamento para a Hipertensão Arterial (HA) e ao Diabetes Mellitus (DM), várias estratégias e ações vêm sendo criadas e também adotadas pelo Ministério da Saúde. Assim, o Plano de Reorganização da Atenção à HA e ao DM merece destaque. São utilizadas estratégias como (reuniões mensais com ações educativas, estímulo à realização de atividades físicas, consultas médicas agendadas e entrega dos medicamentos para estas pessoas). Então, os municípios possuem uma programação ideal para cada local de atividades e para os indivíduos que estão cadastrados no Programa de HA e DM e assim, proporcionar uma atenção maior a esse grupo (Câmara, 2016).

Nesta pesquisa, a grande maioria dos idosos estudados $(46,5 \%)$ realiza atividade física e $(37,2 \%)$ praticam todos os dias. Um número razoável de idosos ainda ingerem bebida alcoólica (16,3\%) e são tabagistas $(9,3 \%)$, mas a grande maioria não apresenta estes maus hábitos de vida (Tabela 4). Os maus hábitos de vida corroboram para uma má saúde. Pessoas praticantes 
de exercícios físicos vão ter uma melhor saúde e uma melhor qualidade de vida, pois, atividade física realizada com regularidade é um dos principais fundamentos para a manutenção da saúde ao longo da vida, assim, evidencia-se que com o passar do tempo o sistema imunológico vai declinando, e com a prática de exercícios físicos, os mesmos colaboram para a redução dos problemas de saúde e a melhor QDV (Fernandes, 2014).

Segundo o estudo realizado por Barbosa et al. (2018), uma pesquisa composta por 400 indivíduos, com a média de idade de 73,8 anos, em sua maioria mulheres $(64,5 \%)$, no que se refere ao consumo de álcool, $(26,7 \%)$ dos idosos ingeriam bebidas alcoólicas e a prevalência do tabagismo foi de $(9,0 \%)$, o que corrobora com esta pesquisa. Em sua pesquisa, o mesmo demonstra que "em 2011, o tabagismo foi responsável por 147.072 óbitos, 2,69 milhões anos de vida perdidos, 157.126 infartos agudos do miocárdio, 75.663 acidentes vasculares cerebrais e 63.753 diagnósticos de câncer".

De acordo com Costa et al. (2017), o abuso de álcool influencia grandemente a morbimortalidade dessas pessoas diante do próprio fator de envelhecimento e ainda é caracterizado como uma epidemia que ocorre de forma silenciosa.

Foi observado que os idosos apresentaram uma boa capacidade funcional e isto, pode ser explicado pelos bons hábitos de vida e a regularidade da pratica de atividade física, tendo déficits apenas em relação à continência, onde (14\%) tem dependência funcional para incontinência urinária.

Segundo Barbosa, et al. (2014), a avaliação da funcionalidade dos idosos nas ESF é de grande importância, e não só o diagnóstico das patologias deve ser incluído, mas também a consciência em relação a funcionalidade desses indivíduos, que envolvem, além da saúde física e mental, as condições socioeconômicas e de capacidade de autocuidado, que revelaram o grau de independência funcional do idoso. No seu estudo, onde foram avaliados 286 idosos, a idade variou de 60 a 103 anos, os idosos precisaram de ajuda principalmente para: controle da micção $(9,4 \%)$, isto evidencia ainda mais esse fator incapacitante dos idosos, com grande repercussão na vida dos mesmos.

"A avaliação da capacidade funcional pode ser uma ferramenta essencial para as Estratégias de Saúde da Família, que presta atendimento aos idosos, para que se tenha ciência da escolha do tipo de intervenção a ser feita e da implementação de ações para cuidados preventivos, preservando ao máximo sua independência e autonomia." (Monteiro et al., 2021)

O envelhecimento fisiológico associa-se ao maior índice para desenvolver doenças (diabetes mellitus, insuficiência cardíaca, entre outras), onde as mesmas podem interferir significativamente na autonomia, na mobilidade, na destreza manual, na lucidez e na capacidade funcional das vias urinárias inferiores e da bexiga da pessoa idosa, favorecendo o aparecimento da Infecção do Trato Urinário (ITU) (Oliveira et al., 2014).

Segundo Carneiro et al. (2017) "Perpetua-se um impacto negativo na qualidade de vida do paciente idoso, pois a ITU representa um importante problema higiênico e social".

Diante da realização da avaliação feita com a Escala de Lawton e de acordo com os escores obtidos, (87\%) dos homens apresentaram independência e (13\%) apresentaram dependência parcial; (86\%) das mulheres são independentes e (14\%) com dependência parcial, dados referentes a (Tabela 6).

Em relação às AIVD's pode-se evidenciar que a maioria dos idosos, são independentes (86\%) com score de 27 pontos, sendo que as mulheres apresentaram em relação aos homens uma maior dependência parcial com score de 26 a 18 pontos (14\%) de acordo com os dados da avaliação.

De acordo com Paula et al. (2013), em sua amostra que foi composta por 120 idosos, as mulheres representaram (66\%) do total, e o grupo de idosos mais velhos com 80 anos ou mais predominou sobre os mais jovens na faixa etária de 60 a 64 anos, com idade mínima de 60 anos e máxima de 94 anos. Não se verificou diferença estatisticamente significativa entre os gêneros no que se refere ao desempenho funcional para as AVD. Mas foi verificada em relação às AIVD uma diferença significativa, tendo maior frequência de idosas que necessitavam de ajuda para realizar essas atividades $(61,25 \%)$ em comparação com os homens que a maioria se apresentou independente, registrando $(\mathrm{p}<0,011)$. 
No estudo de Santos e Cunha (2013), em sua pesquisa realizada com 340 idosos com idade variando entre 60 e 85 anos, no desempenho para AIVD, foi possível observar que apenas $(32,6 \%)$ idosos conseguem usar o telefone sem ajuda. Ao que se refere ao uso de meio de transporte $(33,2 \%)$ realiza de maneira independente. Realizar compras apenas $(32,1 \%)$ idosos consegue sem auxílio. Preparo das refeições é uma atividade que apenas (33,5\%) realizam com independência. Apenas $(35,6 \%)$ dos idosos consegue arrumar a casa, sem ajuda. Lavar roupas é uma tarefa que $(27,4 \%)$ fazem sem auxílio. Trabalhos manuais apenas $(29,1 \%)$ fazem sem auxílio. Tomar os medicamentos em doses e horários corretos somente (25\%) idosos consegue realizar de forma independente. Quanto ao manejo das finanças apenas $(22,9 \%)$ realizam sem auxílio. Evidenciado isto, em relação a esta pesquisa, os idosos apresentaram maior dependência.

Porém, no estudo supracitado, evidencia-se que a maioria dos idosos apresentou incapacidade funcional, mas diante disto, foi observado que em relação a prática de atividade física, $(80,3 \%)$ dos idosos não pratica nenhuma atividade física (Santos \& Cunha, 2013). Na presente pesquisa, $(46,5 \%)$ dos idosos realizam atividade física e $(37,2 \%)$ praticam todos os dias, o que nos leva a crer, que seja um fator de proteção para preservação da funcionalidade.

Referente ao risco para quedas $(23,3 \%)$ dos idosos pesquisados apresentaram $<10$ segundos no teste de caminhada e $(76,7 \%)$ realizaram o teste de 10 a 20 segundos (Tabela 7 ).

Quanto ao risco de quedas, 33 idosos (76,7\%) apresentaram médio risco para quedas. Para Tinetti (2003 apud Dantas; Brito; Lobato, 2012) esclarece que todos os anos mais de um terço das pessoas com 65 anos ou mais sofrem episódios de quedas no mundo, tendo possibilidade de recorrência de quedas em metade dos casos.

Segundo Dantas et al. (2012), na população idosa, a ocorrência das quedas pode resultar em varias consequências que ocasionam prejuízos tanto físicos e psicológicos, quanto econômicos devido à atenção médico-social, apontados pela utilização de vários serviços especializados e, especialmente, pelo crescimento do número de hospitalizações, um fato que é discutido em vários estudos, a prevalência das quedas nos idosos, estes fatores aliados ao declínio funcional nas AVD, levam à redução da mobilidade e independência, o que pode resultar em processo de deterioração física e propensão ao evento fatal.

De acordo com Souza et al. (2017), em seu estudo realizado com 22 idosos com a idade variando entre 60 a 84 anos, sendo 16 do sexo feminino e 6 do masculino na UBS do município de Guanambi-BA, ao realizar o teste TUGT, os idosos apresentaram um risco de quedas médio $(63,7 \%)$. Sendo do sexo feminino $(45,5 \%)$ e sexo masculino $(18,2 \%)$. O que está de acordo com a presente pesquisa.

Foi evidenciado que dos 43 idosos entrevistados, $(65,1 \%)$ correspondem ao sexo feminino, evidenciando que existe uma maior acessibilidade de mulheres na UBSF.

No estudo realizado por Ferrari, et al. (2014), os resultados mostraram que na análise em 106 prontuários, $(73,58 \%$ ) são de idosas do sexo feminino, configurando maior procura da unidade de saúde por parte das mulheres. Em outro estudo, realizado por Melo; Ferreira; Teixeira (2014) evidencia-se que "a esperança de vida feminina é superior à masculina, sendo 77 anos e 69 anos, respectivamente, o que torna as mulheres mais vulneráveis". O mesmo, em seu estudo apresentou a média de idade dos idosos pesquisados que foi de aproximadamente $70( \pm 8)$ anos, sendo que a idade mínima foi de 60 anos e, a máxima, de 104 anos, visto que $(54,2 \%)$ eram do sexo feminino.

Em relação à avaliação da escala de Katz, referente ao sexo (Gráfico 1), sobre o quesito continência, (21\%) apresentaram-se com dependência funcional para incontinência urinária e as mulheres são afetadas neste estudo. Segundo Marques (2016), na literatura evidencia-se que os estudos mais recentes têm demonstrado o impacto que a infecção do trato urinário (ITU) causa nas atividades ocupacionais da vida, chamando a atenção dos pesquisadores, visto que os resultados têm mostrado que a ITU traz consequências tanto na vida pessoal como no desempenho profissional destas mulheres, causando um grande impacto na QDV das mesmas. 
Diante das alterações que acontecem com as idosas, o estrogênio e progesterona são os hormônios femininos que desempenham importantes papéis no corpo da mulher, e com a velhice, os mesmos passam a sofrer alterações, a queda dos níveis destes pode causar uma porção de sintomas físicos e emocionais, que inclui ondas de calor, alterações de humor e secura vaginal (Marques, 2016).

A incontinência urinária em mulheres é em torno de (58,8\%) e (39,2\%) entre os homens. As mulheres então, ao decorrer de sua vida, são mais vulneráveis para adquirir infecções do trato urinário o que se justifica as diferenças que ocorrem entre o homem e mulher na fase de envelhecimento humano (Santos \& Bianchi, 2014).

Referente ao Gráfico 2 na avaliação da escala de Lawton, as mulheres apresentaram em relação aos homens uma maior dependência parcial com score de 26 a 18 pontos de acordo com os dados da avaliação.

Nesta pesquisa, os idosos que tiveram dependência parcial, apresentaram dificuldades nas atividades de: usar o telefone, viajar, cozinhar, arrumar a casa, trabalhos domésticos, lavar e passar a roupa.

No estudo realizado com 117 idosos com idade igual ou superior a 80 anos, na zona urbana do município de Jequié/BA, com idosos residentes em domicílio, cadastrados em cinco Unidades de Saúde da Família (USF), (80,3\%) se mostraram dependentes em relação às AIVD, e foi observado que a maioria da população estudada foi do sexo feminino $(59,0)$, semelhante a diversos estudos realizados com população longeva (Novais et al., 2016).

No teste de TUGT (Gráfico 3), foi evidenciado que a maioria desses idosos apresentaram médio risco para quedas e as mulheres se apresentaram com maior prevalência (82\%).

Rossi (2008), fala que a perda de massa óssea (osteopenia) por involução ocorre, com prevalência, no sexo feminino pós-menopausada, quando a falta do freio estrogênico libera a voracidade dos osteoclastos. Evidencia-se que após os 60 anos de idade, ocorre uma prevalência de perda da massa muscular, chamada de (sarcopenia) seja da ordem de (30\%) e aumentando progressivamente com o envelhecimento fisiológico.

As mulheres apresentaram uma prevalência de osteoporose muito maior em relação aos homens e a menopausa é um fator diretamente ligado para este acontecimento, prevalecendo assim, o aumento do risco de fraturas (Rodrigues \& Barros, 2016).

\section{Conclusão}

Os resultados do presente estudo fornecem considerações relevantes sobre o perfil de idosos atendidos na atenção básica da cidade de pocinhos, interior da paraíba.

Observou-se por meio deste estudo que a maioria dos idosos da amostra, encontram-se na faixa etária de 60 a 69 anos, com predominância do sexo feminino de $(65,1 \%)$. Com relação à raça $(62,8 \%)$ são de cor branca. Ainda dentro da amostra estudada $(62,8 \%)$ são casados e $(90,7 \%)$ tem filhos. Já de acordo com a escolaridade $(69,7 \%)$ apresentam baixa escolaridade, tendo apenas o ensino fundamental incompleto. Pode-se também concluir que $(58,1 \%)$, recebem até um salário mínimo, enquadrando-se de acordo com o estudo como renda familiar baixa, boa parte da população da amostra reside com alguém e $(18,6 \%)$ relataram morar sozinhos. Uma parcela considerável do estudo que compreende $(95,3 \%)$ recebem assistência de médico, (93\%) enfermeiro e $(81,3 \%)$ do agente comunitário de saúde.

Grande parte dos idosos deste estudo apresentam-se independentes tanto para as ABVD's quanto para as AIVD's, considerando que (46,5\%) praticam alguma atividade física e a maior porcentagem não fuma e nem ingere bebidas alcoólicas, nos possibilitando compreender, de acordo com o estudo, que estes idosos possuem bons hábitos de vida, fato que contribui para melhor qualidade de vida dos mesmos. 
Foi possível observar que estes idosos em relação a outros estudos, apresentam uma faixa etária com predominância de 60 a 69 anos, o que contribui para a melhor capacidade funcional evidenciada nos dados obtidos durante a pesquisa. Mas, quanto ao risco de quedas, a maior parte apresentou médio risco.

A pesquisa também pode evidenciar que $(76,7 \%)$ possui alguma doença crônica, e a de maior prevalência encontrada foi a Hipertensão Arterial Sistêmica com um percentual de (58,1\%) e em segundo a Diabetes, atingindo $(18,6 \%)$.

O sexo feminino teve maior predominância, dado que evidencia que as mulheres tem uma maior preocupação com a saúde. Um ponto importante deste trabalho que explica a menor procura dos homens por atendimento de saúde na UBSF se dá pelo fato de que suas esposas quando veêm as consultas, acabam pegando as receitas de seus respectivos esposos ou de algum familiar.

Foi possível identificar a diferença funcional entre os sexos, mesmo com um número de mulheres maior que o de homens, demonstrando que as mulheres apresentaram maior dependência em relação aos homens desse estudo, principalmente, no que diz respeito à continência urinária.

Por fim, todos os objetivos impostos nesta pesquisa, foram alcançados com clareza e ao observar todos esses achados, seria de suma importância, aplicar um protocolo de exercícios fisioterapêuticos para estes idosos, realizando a comparação, quanto a avaliação do teste de TUGT, visto que foi evidente o médio risco desses idosos para sofrer quedas, e assim, possibilitar melhor qualidade de vida e funcionalidade para estes indivíduos.

Diante disto, podemos concluir que a pesquisa realizada trouxe inúmeros benefícios para a população estudada e para a população ouvinte. Visando esse contexto, pode-se analisar uma possível sequência evolutiva deste estudo, tomando como amostra não apenas os idosos que foram até a UBS, mas sim, todos os idosos cadastrados na UBS a qual o estudo foi realizado, proporcionando um estudo mais amplo e resultados mais fidedignos.

\section{Referências}

Alves, J. E. D. (2014). Transição demográfica, transição da estrutura etária e envelhecimento, Revista portal de divulgação, 8-15. https://revistalongeviver.com.br/index.php/revistaportal/article/view/440/440

Barbosa, B. R. et al. (2014). Avaliação da capacidade funcional dos idosos e fatores associados à incapacidade, Ciênc. saúde colet, 19(8), 3317-3325. https://www.scielo.br/j/csc/a/hcBn67RFRt3brvSNp5YsDFh/abstract/?lang=pt

Barbosa, M. B. et al. (2018). Prevalência e fatores associados ao consumo de álcool e de tabaco em idosos não institucionalizados, Rev. Bras. Geriatr. Gerontol, 21(2), 125-135. https://www.scielo.br/j/rbgg/a/hsRjGdPqSWMFTrzsGhFSXfq/?lang=pt

Câmara, F. T. (2016). O processo organizacional dos grupos de hiperdia e saúde mental em uma UBS: Um modelo de intervenção, Una-sus, 6-18. https://ares.unasus.gov.br/acervo/html/ARES/7902/1/Fernanda\%20Teixeira\%20C\%C3\%A2mara.pdf

Carneiro, J. A. et al. (2017). Prevalência e fatores associados à incontinência urinária em idosos não institucionalizados, Ciênc. saúde colet, 25(3), 268-277. https://www.scielo.br/j/cadsc/a/qNqQKxfzV3qV6y65cGvWd3M/abstract/?lang=pt

Cazella, A. A. et al. (2016). Políticas Públicas de Desenvolvimento Rural no Brasil: o dilema entre inclusão produtiva e assistência social, Política \& Sociedade, $1(15), 49-79$.

Chaimowicz, F. et al. (2013). Saúde do idoso, Una-sus, 1-182. https://ares.unasus.gov.br/acervo/handle/ARES/2011

Cockell, F. F. (2014). Idosos aposentados no Mercado de trabalho informal: trajetórias ocupacionais na construção civil, Psicologia \& Sociedade, 26(2), 461471.

Costa, C. M. F. N. et al. (2017). Utilização de medicamento pelos usuários da atenção primária do Sistema Único de Saúde, Rev Saude Publica, 1(51), 1-18.

Costa, I. P. et al. (2017). Aspectos relacionados ao abuso e dependência de álcool por idosos, Rev Enferm Ufpe On Line, 11(6), $232-238$.

Dantas, E. L, Brito, G. E. G \& Lobato, I. A. F. (2012). Prevalência de quedas em idosos adscritos à estratégia de saúde da família do município de joão pessoa, paraíba, Rev Aps, 15(1), 67-75.

Dias, D. S. G, Carvalho, C. S \& Araujo, C. V. (2013). Comparação da percepção subjetiva de qualidade de vida e bem-estar de idosos que vivem sozinhos, com a família e institucionalizados, Revista Brasileira de Geriatria e Gerontologia, 16(1), 127-138.

Esquenazi, D, Silva, S. R. B \& Guimarães, M. A. M. (2014). Aspectos fisiopatológicos do envelhecimento humano e quedas em idosos, Revista Hupe, 13(2), 
11-20.

Fernandes, B. L. V. (2014). Atividade Física no processo de envelhecimento, Revista Portal de Divulgação, (40), $43-48$.

Ferrari, R. F. R. et al. (2014). Motivos que levaram idosos com hipertensão arterial a procurar atendimento na atenção primária, Rev Rene, 15(4), 691-700.

Lopes, M. J. et al. (2013). Avaliação da funcionalidade e necessidades de cuidados dos idosos, Rev. Latino-Am. Enfermagem, 1-9.

Magri, S, et al. (2020). Programa de educação em saúde melhora indicadores de autocuidado em diabetes e hipertensão, Rev Eletron Comun Inf Inov Saúde, $14(2), 386-400$.

Marques, S. R. Tratamento fisioterapêutico na incontinência urinária em idosas, Revista Saúde Integrada, 9(17), $110-116$.

Martins, R. M. L \& Mestre, M. A. (2014). Esperança e qualidade de vida em idosos, Millenium, 153-162. http://repositorio.ipv.pt/bitstream/10400.19/2620/1/13.pdf.

Melo, N. C. V, Ferreira, M. A. M \& Teixeira, K. M. D. Condições de vida dos idosos no brasil: uma análise a partir da renda e nível de escolaridade, Revista Brasileira de Economia Doméstica, 25(1), 004-019.

Miranda, G. M. D, Mendes, A. C. G \& Silva, A. L. A. (2016). O envelhecimento populacional brasileiro: desafios e consequências sociais atuais e futuras, Rev. Bras. Geriatr. Gerontol, 19(3), 507-519.

Monteiro, A.E, Pereira, H.S \& Amorim, P.B. (2021). avaliação da capacidade de idosos através da análise do índice de barthel, Revista científica multidisciplinar, 2(9), 1-11.

Novais, M. M. et al. (2016). Avaliação de indicadores de desempenho funcional de idosos longevos residentes em domicílio, Arq. Ciênc. Saúde, 23(3), 67-72.

Oliveira, A. S. et al. (2014). Fatores ambientais e risco de quedas em idosos: revisão sistemática, Rev. Bras. Geriatr. Gerontol, 17(3), 637-645.

Paula, A. F. M. et al. (2013). Avaliação da capacidade funcional, cognição e sintomatologia depressiva em idosos atendidos em ambulatório de Geriatria, Rev Bras Clin Med, 11(3), 212-218.

Pascotini, F. S. et al. Força muscular respiratória, função pulmonar e expansibilidade toracoabdominal em idosos e sua relação com o estado nutricional, Fisioter Pesqui, 23(4), 416-422.

Pimentel, I \& Scheicher, M. E. Comparação da mobilidade, força muscular e medo de cair em idosas caidoras e não caidoras, Rev. Bras. Geriatr. Geronto, 6(2), 251-257.

Prodanov, C. C \& Freitas, E. C. (2013). Metodologia do trabalho ciêntifico: Métodos e técnicas da pesquisa e do trabalho acadêmico https://books.google.com.br/books?hl=pt-BR\&lr=\&id=zUDsAQAAQBAJ\&oi=fnd\&pg=PA13\&dq=Tipos+de+pesquisa+cientifica\&ots=db-

1cdA7EQ\&sig=BI698t8iAycKodAAZU-rHfLaErc\#v=onepage \&q \&f=false

Puerro Neto, J \& Brito, C. A. F. (2015). Mobilidade funcional em função da força muscular em mulheres idosas fisicamente ativas, Rev Bras Med Esporte, 21(5), 369-371.

Ramos, L.R. et al. (2013). Perguntas mínimas para rastrear dependência em atividades da vida diária em idosos, Rev Saúde Pública, 47(3), 506-513.

Resende Neto A. G, Silva, G. M. E, Santos, M. S \& Cyrino, E. S. Treinamento funcional para idosos: uma breve revisão, R. bras. Ci. e Mov, $24(3)$, 167-177.

Reis, L. A; Rocha, T. S, Duarte, S. F. P. (2014). Quedas: Risco e fatores associados em idosos institucionalizados. Revista Baiana de Enfermagem, 28(3), 225234.

Rossi, E, et al. (2008). Envelhecimento do sistema osteoarticular, Einstein, 6(1), 7-12.

Santos, G. S \& Cunha, I. C. K. (2013). O. Avaliação da capacidade funcional de idosos para o desempenho das atividades instrumentais da vida diária: um estudo na atenção básica em saúde, R. Enferm. Cent. O. Min, 3(3), 820-828.

Santos, D. C. A, Bianchi, L. R. O. (2014). Envelhecimento Morfofuncional: diferença entre os gêneros, Arquivos do Mudi, 18(2), 33-46.

Silva, V. R, Souza, G. R \& Alves, S. C. C. (2015). Benefícios do exercício físico sobre as alterações fisiológicas, aspectos sociais, cognitivos e emocionais no envelhecimento, Revista Cpaqv, 7(3), 2-12.

Silva, A \& Prá, K. R. D. (2014). Envelhecimento populacional no Brasil: o lugar das famílias na proteção aos idosos. Argumentum, 6(1), 99-115.

Silva, J.V.F. et al. (2015). A relação entre o envelhecimento populacional e as doenças crônicas não transmissíveis: sério desafio de saúde pública, Ciências Biológicas e da Saúde, 2(3), 91-100.

Silva, T. O. et al. (2014). Grupo de assistência multidisciplinar ao idoso ambulatorial: efeitos da abordagem fisioterapêutica, Rev Soc Bras Clin Med, 12(1), 2 7.

Silva, F. O, Suto, C. S. S \& Costa, L. E. L. (2015). Perfil de pacientes cadastrados no hiperdia: conhecendo o estilo de vida, Rev. Saúde Col. Uefs, 5(1), 33-39.

Souza, L. H. R. et al. (2017). Queda em idosos e fatores de risco associados, Rev. Aten. Saúde, 15(54), 55-60.

Souza, C. (2017). Teste Timed Up and Go http://kinetec.com.br/blog-detalhe-tecnologia-biomecanica/teste-timed-up-and-go/20

Xavier, P. B, et al. (2019). alterações fisiológicas/patológicas do envelhecimento e seu potencial repercurssor sobre o risco de queda, Cieh, 1-9. https://editorarealize.com.br/editora/anais/cieh/2019/TRABALHO_EV125_MD1_SA2_ID198_29042019233637.pdf 